\title{
High Resolution Software Defined Radar System for Target Detection
}

\author{
S. Costanzo, ${ }^{1}$ F. Spadafora, ${ }^{1}$ A. Borgia, ${ }^{1}$ H. O. Moreno, ${ }^{2}$ A. Costanzo, ${ }^{1}$ and G. Di Massa ${ }^{1}$ \\ ${ }^{1}$ Dipartimento di Ingegneria Informatica, Modellistica, Elettronica e Sistemistica, Università della Calabria, 87036 Rende, Italy \\ ${ }^{2}$ Facultad de Informatica y Electronica, Escuela Superior Politecnica de Chimborazo, Riobamba EC060155, Ecuador \\ Correspondence should be addressed to S. Costanzo; costanzo@deis.unical.it
}

Received 10 May 2013; Revised 14 July 2013; Accepted 25 August 2013

Academic Editor: Alvaro Rocha

Copyright (c) 2013 S. Costanzo et al. This is an open access article distributed under the Creative Commons Attribution License, which permits unrestricted use, distribution, and reproduction in any medium, provided the original work is properly cited.

\begin{abstract}
The Universal Software Radio Peripheral USRP NI2920, a software defined transceiver so far mainly used in Software Defined Radio applications, is adopted in this work to design a high resolution L-Band Software Defined Radar system. The enhanced available bandwidth, due to the Gigabit Ethernet interface, is exploited to obtain a higher slant-range resolution with respect to the existing Software Defined Radar implementations. A specific LabVIEW application, performing radar operations, is discussed, and successful validations are presented to demonstrate the accurate target detection capability of the proposed software radar architecture. In particular, outdoor and indoor test are performed by adopting a metal plate as reference structure located at different distances from the designed radar system, and results obtained from the measured echo are successfully processed to accurately reveal the correct target position, with the predicted slant-range resolution equal to $6 \mathrm{~m}$.
\end{abstract}

\section{Introduction}

Radar systems have been employed for a long time mainly in military operation, like target detection, target recognition, surveillance, and other specific applications, such as meteorology and air-traffic control. However, especially in the last recent years, new kind of large-scale commercial applications is requiring the standard radar system operations, but according to significant cost reduction and strong adaptability. Medical diagnostics and automotives are just some examples of the possible application fields. According to this new operating context, Software Defined Radar (SDRadar) could represent a new challenge in radar technology due to the possibility of performing most of the basic operations (i.e., mixing, filtering, modulation, and demodulation) by simply employing software modules in order to strike out most of the specific hardware [1]. The main goal of a software defined approach is related not only to a clear cost reduction, but also to a significant increase of the versatility of the system, since signal generation and signal processing parameters may be easily adapted on the fly to the task under consideration. New researches are recently conducted to implement a SDRadar for target distance detection $[2,3]$, but they are based on the use of sophisticated FPGA and/or DSP, thus being not able to guarantee the required cost reduction.

A possible solution for a low cost SDRadar system development can be obtained by the adoption of the Universal Software Radio Peripheral (USRP) transceiver. A first attempt to apply USRP for target detection is performed in [4]; however, due to the bandwidth limitations imposed by the available USB connection, a limited slant-range resolution equal to $75 \mathrm{~m}$ is obtained. Interesting results are presented in [5-8], where different characterizations of the USRP N200 e N210 are employed as SDRadar systems. However, these transceivers are not able to easily design a compact device; moreover, a full adaptability of the software code on the fly for different scenarios can be scarcely achieved. National Instruments (NI) has recently manufactured two new generation devices, the USRP NI2920 and USRP NI2921, successfully combining the NI LabVIEW software and the USRP hardware to deliver an affordable and easy-to-use software-reconfigurable RF platform potentially suitable for experimentation, research, and rapid prototyping [9]. These devices, as yet used for teaching, communication, and mainly 


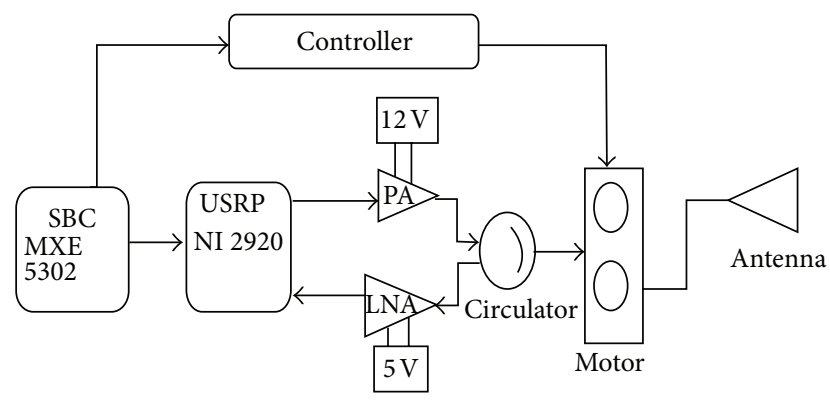

FIGURE 1: Block diagram of the proposed SDRadar system.

in Software Defined Radio applications [10], are considered in this work as a potential transceiver for a compact and versatile SDRadar. In particular, as described in Section 2, USRP NI2920 is employed in a SDRadar design for target detection.

Since one of the most relevant problems of SDRadar developing is related to a low slant-range resolution, due to the low speed of its interface, the main goal of this work is to explore the potentialities of the NI new generation USRP in order to enhance this specific parameter, taking advantage of the Gigabit Ethernet Interface. A LabVIEW code is developed to both control the features of the SDRadar system as well as to perform a signal processing compression technique (not completely suitable for the first generation USRP interface capabilities), thus achieving a strongly enhanced slant-range resolution with respect to the previous systems. Furthermore, experimental indoor and outdoor tests are performed in order to validate the system behavior in terms of target detection capability. A possible application scenario of the designed L-Band SDRadar system can be represented by the landslides monitoring in the presence of vegetated areas.

\section{Proposed SDRadar System}

The software radar architecture proposed in this work is developed to create a low cost, compact, and flexible solution for an accurate target detection; potentially, this scheme can be easily adopted in different scenarios without significant hardware modification, thus leading to create a multipurpose radar system. The complete block diagram of the proposed SDRadar system is reported in Figure 1. The USRP 2920 platform is used to transmit and receive data by a linear array antenna with specific bandwidth and directivity features useful for the prescribed application [11-16].

One of the main motivations for the choice of the USRP NI2920 is due to the enhanced available bandwidth $(25 \mathrm{MHz})$, when compared to the first version $(2 \mathrm{MHz})$, with the possibility to significantly enhance the radar slant-range resolution. The whole system is controlled by a LabVIEW application running on a compact single board computer (SBC). It is able to perform the required processing for target detection, but it is designed also to control a motor system guaranteeing the antenna, thus giving a scanning feature useful for an accurate surface monitoring in different directions. A power amplifier (PA) and a low noise amplifier
(LNA) increase the signal power along both the transmission and the receiving paths, and a circulator can also be adopted to use a single antenna in both the transmission and the receiving paths, thus further reducing the total hardware costs and the size of the entire device.

\section{Signal Processing Algorithm}

A stretch processor (SP) approach [17], according to the diagram scheme of Figure 2, is adopted to implement the signal processing stage. First, the radar echo signal is mixed with a replica (reference signal) of the transmitted waveform. Then, a low pass filtering (LPF) and a coherent detection are performed in order to avoid the high frequency response coming from the mixer output. Analog to digital (A/D) conversion is subsequently applied, and a bank of narrow band filters is adopted to extract the tones proportional to the target range. Finally, a Fast Fourier Transform (FFT) is applied to derive the amplitude spectrum, whose peaks are related to the targets positions.

The adopted transmitted signal is a linear frequency modulated waveform expressed by the following equation:

$$
s(t)=\cos \left[2 \pi\left(f_{0} t+\frac{\mu}{2} t^{2}\right)\right], \quad 0<t<\tau^{\prime},
$$

where $\mu=B / \tau^{\prime}$ is the LFM coefficient, $B$ gives the bandwidth, $f_{0}$ is the chirp start frequency, and $\tau^{\prime}$ is the chirp duration.

The slant-range resolution $\Delta R$ is then provided by the expression [17]

$$
\Delta R=\frac{c}{2 B}
$$

thus being strictly related to the system bandwidth $B$.

As imposed by (2), the use of USRP NI 2920 offers a maximum available bandwidth of $25 \mathrm{MHz}$, leading to have a slant-range resolution $\Delta R=6 \mathrm{~m}$, which is significantly enhanced when compared to the value $\Delta R=75 \mathrm{~m}$ achieved with the first generation USRP, having a bandwidth $B=$ $2 \mathrm{MHz}$ [4].

On the basis of the outlined SP technique, a sophisticated algorithm implementing both the signal processing code and the motor control operations is developed in LabVIEW code (Figure 3) to obtain the proposed scanning SDRadar in Figure 1. In particular, the radar system is designed to perform $\mathrm{N}$ distinct scannings on different frames of the area under analysis (e.g., mountain, landslide, topography surfaces, and glaciers) through the controlled rotation of the radar antenna (Figure 4).

The main steps of SDRadar algorithm, whose block diagram is given in Figure 5, can be summarized as follows.

(1) Parameters definition:

(i) Footprint (antenna illuminating area) of each scan, defined in terms of distance between the radar antenna and the analyzed area, azimuth and elevation antenna beamwidths, grazing angle, and operating frequency; 


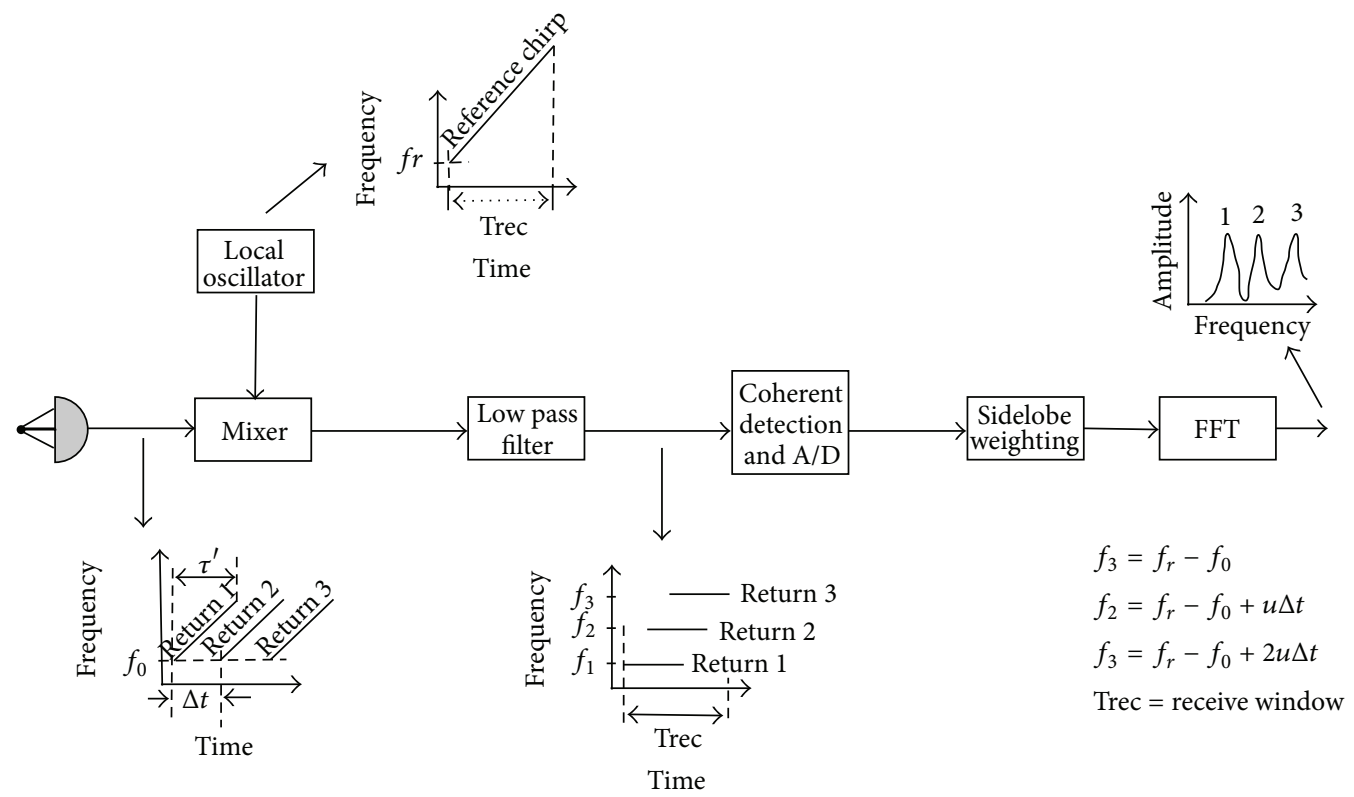

FIGURE 2: Block diagram of the Stretch Processor technique.

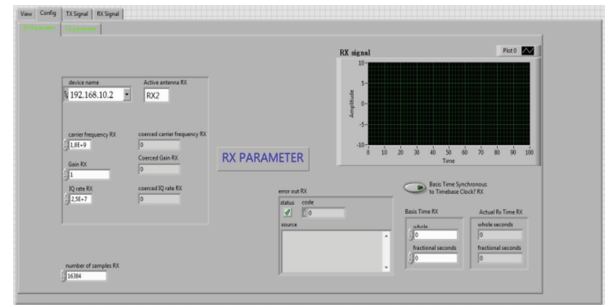

FIGURE 3: LabVIEW SDRadar application window.

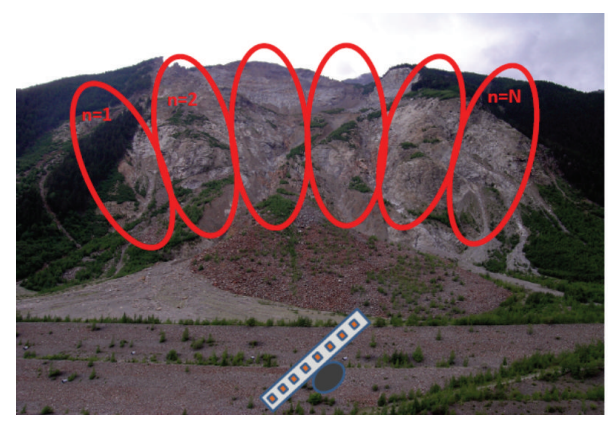

FIGURE 4: Schematic view of SDRadar antenna scanning.

(ii) Receiving window that ensures the correct recognition of any type of topology relative to the surface under analysis, defined by $R_{\min }$ and $R_{\max }$ (minimum and maximum required target range);

(iii) Total area size, giving the exact number $\mathrm{N}$ of radar scannings necessary to retrieve the total topology.
(2) Matrix definitions:

The Matrix is composed by $N$ rows, corresponding to the $N$ produced scanning, and $M$ columns, depending on the receiving angular windows.

(3) Main loop implementing the SP algorithm and the motor control operations.

Once completing the matrix, a color assignment is performed like in a radar gram [18]. Colors are helpful for the remote view of the overall topology.

\section{Experimental Validations}

The performance of the SDRadar system proposed in Section 2 is tested in order to verify the proper distance detection between the radar device and a test metal plate, with dimensions equal to $1.22 \mathrm{~m} \times 0.91 \mathrm{~m}$, placed orthogonally to the direction of propagation of the radar transmitted waves. In this particular scenario, antennas are linearly polarized and the operating central frequency is equal $1.8 \mathrm{GHz}$, so the device operates as an L-band radar.

Specific outdoor tests are performed on the basic system, composed by a PC, USRP 2920, and two antennas, in order to validate the correct behavior of the transceiver in this scenario, and further indoor tests are performed on the complete system, described in Figure 1, with the adoption of the same test target, in order to confirm the enhanced radar resolution.

4.1. Outdoor Tests. A scheme of the outdoor test setup is reported in Figure 6. A broadband ridged horn is adopted as transmitting antenna, while a broadband logarithmic 


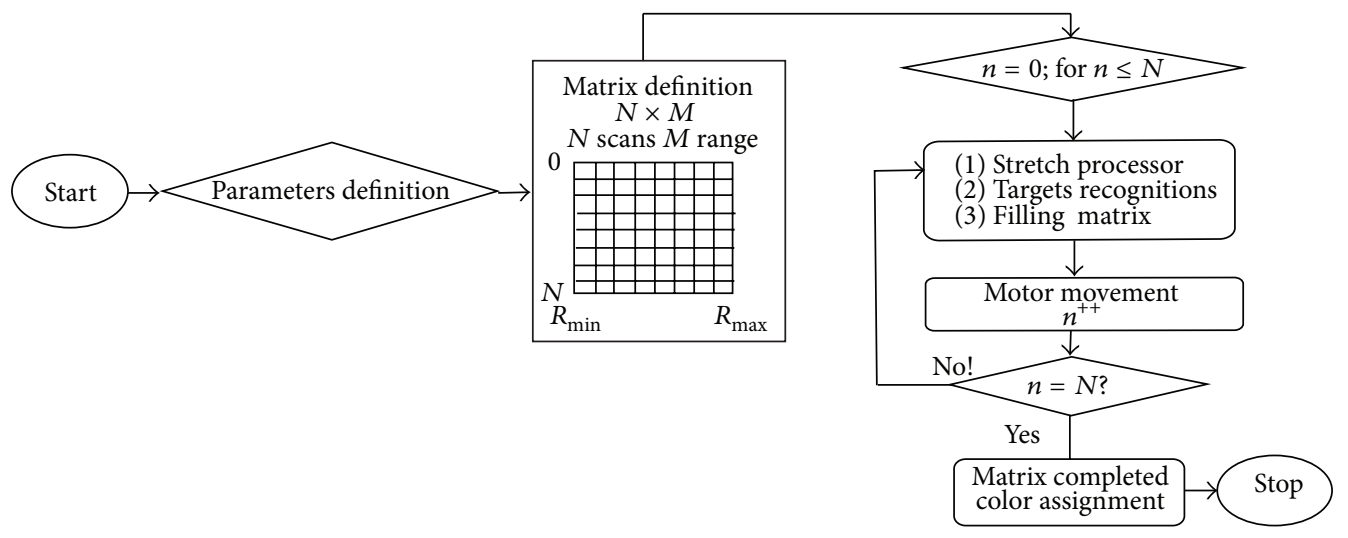

FIGURE 5: Block diagram of the scanning SDRadar algorithm.
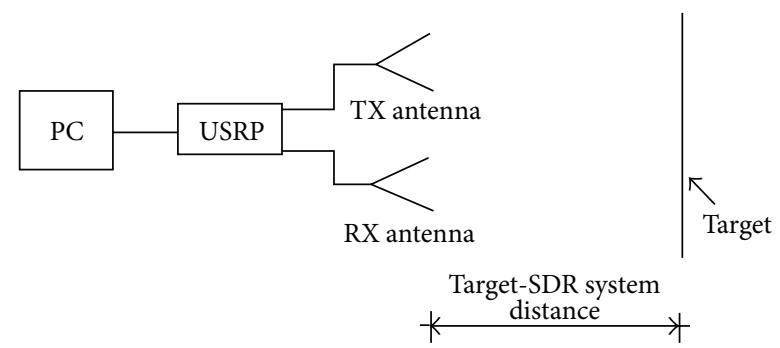

FIGURE 6: Scheme of the basic system for outdoor test.

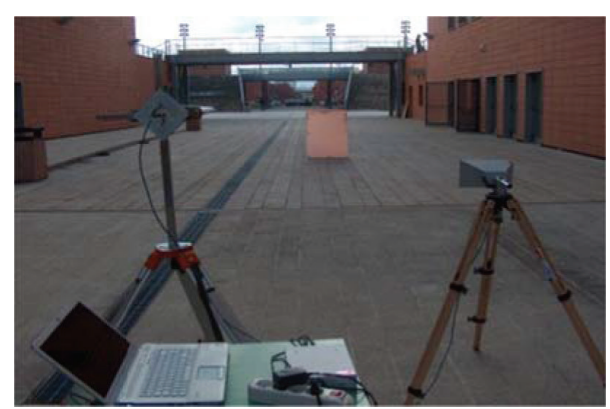

Figure 7: Photograph of outdoor test setup.

antenna, with the same linear polarization, is used in the receiving path.

With reference to the general block diagram of Figure 1, the circulator, the amplifiers, and SBC are excluded in this first validation case, in order to test only the USRP behavior in terms of radar capabilities. A calibration step is preliminarily performed in the absence of the test target, in order to characterize and subsequently remove the undesired reflections due to the open measurement environment, otherwise producing "false" peaks. The test metal plate is subsequently placed at three different reference distances $(6 \mathrm{~m}, 12 \mathrm{~m}$, and $18 \mathrm{~m}$ ) from the transmitting/receiving platform, in order to test the SDRadar slant-range resolution. A noncongested pedestrian zone (Figure 7) is chosen as test location, in order to have negligible contribution of multipath. The results of the FFT processing on the receiving echo for different target
TABLE 1: Real and retrieved target positions with the NI-USRP 2920.

\begin{tabular}{lc}
\hline Real target position $[\mathrm{m}]$ & Retrieved target position $[\mathrm{m}]$ \\
\hline $0 \div 6$ & 6 \\
$6 \div 12$ & 12 \\
$12 \div 18$ & 18 \\
\hline
\end{tabular}

positions are illustrated in Figure 8, while a comparison between the true and the software retrieved target positions is summarized in Table 1.

The results relative to the reconstruction of the three targets positions are represented in terms of SDRadar map described in Section 4, but assuming for simplicity $N=1$ (single scan). The corresponding radar gram, reported in Figure 9, properly displays the targets positions at $6 \mathrm{~m}, 12 \mathrm{~m}$, and $18 \mathrm{~m}$, with three different colors in a gray scale.

The results shown in Figures 8 and 9 confirm the proper distance detection capabilities and a resolution equal to $6 \mathrm{~m}$, thus being 12 times less with respect to that obtained by using the first generation USRP in a SDRadar system.

4.2. Indoor Tests. Similar measurements are performed into the anechoic chamber of the Microwave Laboratory at University of Calabria, in order to test the correct behavior of the entire compact system illustrated in Figure 1. A broadband logarithmic antenna is adopted as both transmitting and receiving device, so the two paths are separated through the use of a circulator. A scheme of the relative test setup is shown in Figure 10. As in the outdoor tests, a preliminary calibration step is performed to avoid false peaks due to reflections in the path between circulator and antenna, power amplifier and circulator, and USRP and power amplifier. Again, a copper sheet is assumed as reference target and positioned at various distances, multiple of the theoretical range resolution, equal to $6 \mathrm{~m}$.

The results of FFT performed on the received signal are illustrated in Figures 11 and 12 for target positions equal to $6 \mathrm{~m}$ and $12 \mathrm{~m}$, respectively.

In both cases, the relative amplitude peaks can be properly distinguished in the diagrams, thus confirming the correct value of the slant-range resolution. 


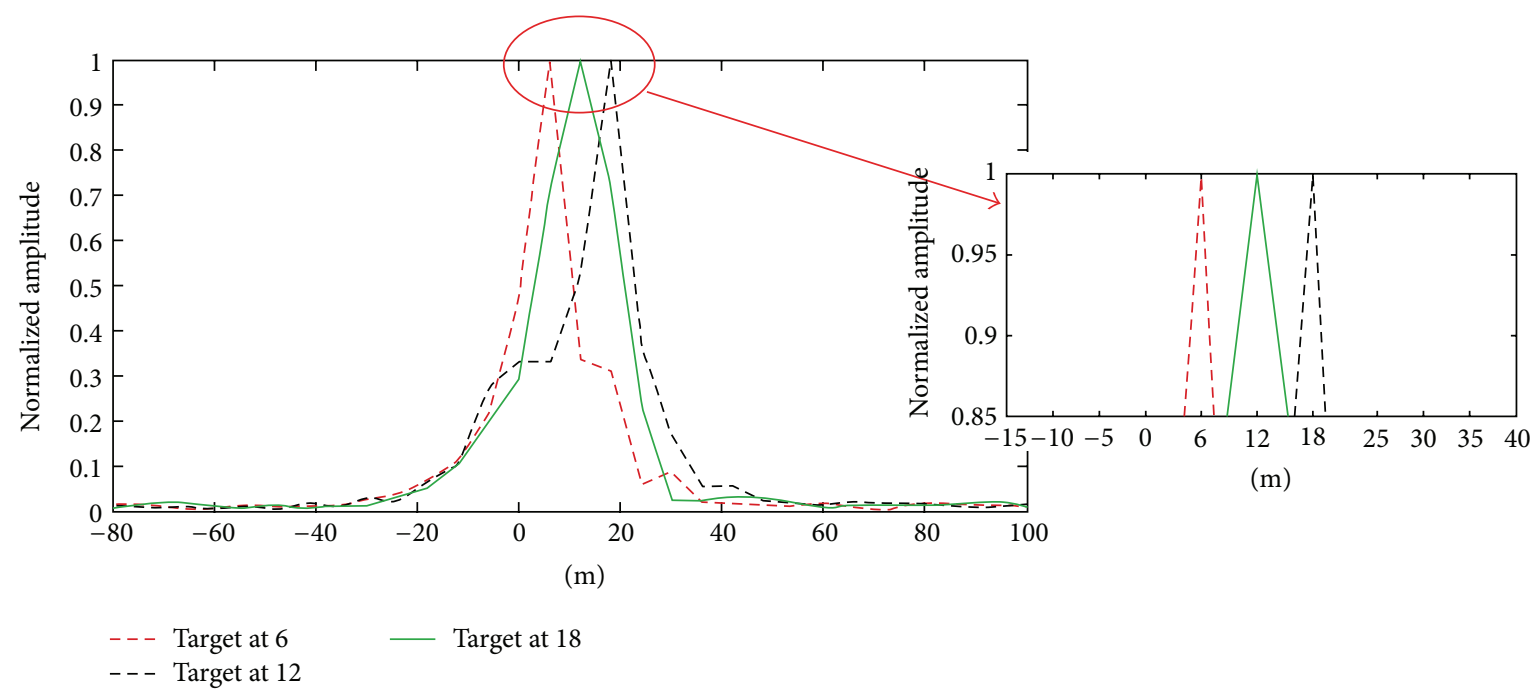

FIGURE 8: Retrieved signal peaks for different target positions.

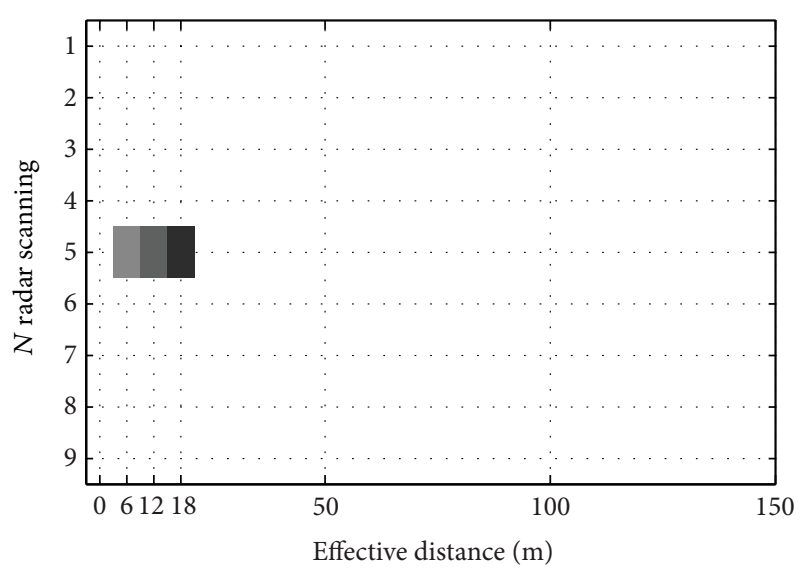

FIGURE 9: SDRadar map for a single scanning.

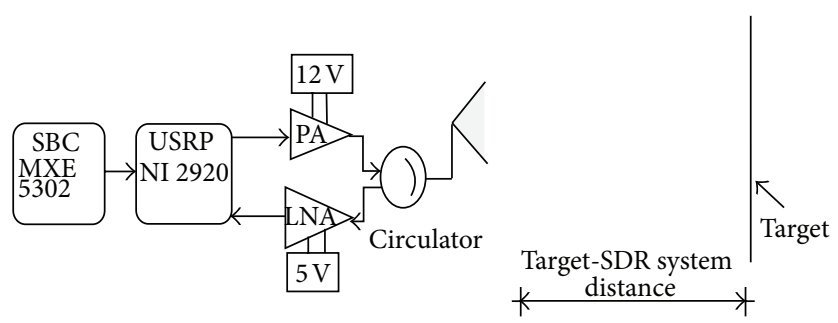

FIGURE 10: Scheme of indoor test setup.

Finally, two identical metal plates are both placed in the test zone, the first one at a distance between $0 \mathrm{~m}$ and $6 \mathrm{~m}$ and the second one between $6 \mathrm{~m}$ and $12 \mathrm{~m}$.

Both relative target positions are accurately identified by the two main peaks corresponding to $6 \mathrm{~m}$ and $12 \mathrm{~m}$ (Figure 13). Other lower peaks, in correspondence with higher distances multiple of the slant-range resolution, represent multipaths of the radar signal between the two metallic plates. However, the low signal corresponding to multipaths does

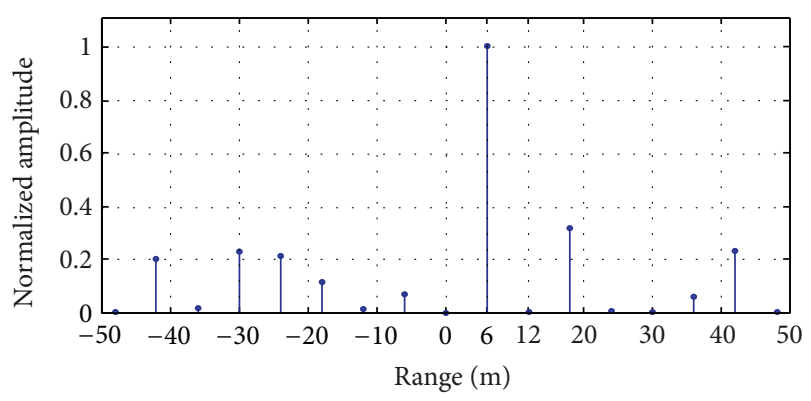

FIGURE 11: Retrieved signal peaks with the target placed at $6 \mathrm{~m}$.

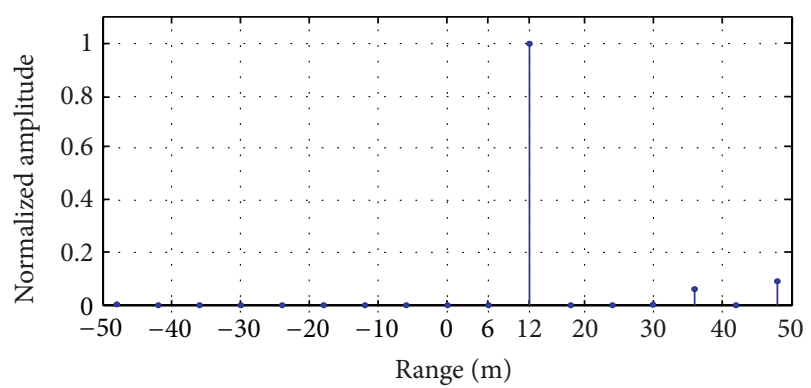

FIGURE 12: Retrieved signal peaks with the target placed at $12 \mathrm{~m}$.

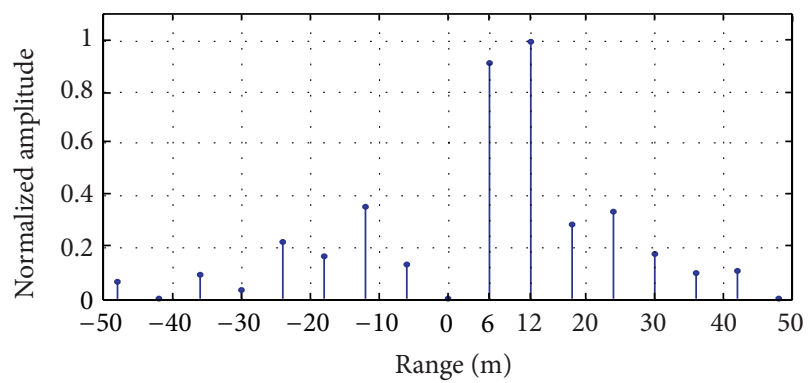

FIGURE 13: Retrieved signal peaks with two targets placed between 0 and $12 \mathrm{~m}$. 
not prevent the correct interpretation of the scene, so indoor tests on the entire compact device confirm the validity of proposed approach.

\section{Final Discussion}

The strong limitation in terms of slant-range resolution, due to the low data rate available at the interface of the first generation USRP and the low adaptability of similar transmitterreceiver devices into a compact device, strongly prevent the potential use of SDRadar in several new applications; as a consequence of this, the resolution enhancement, along with compactness and low costs, is becoming key factor in the SDRadar research. In this work, the use of the new generation NI 2920 USRP has been investigated to design a compact and low-cost SDRadar system able to provide appealing slantrange resolutions with a strong improvement (from $12 \mathrm{~m}$ to $6 \mathrm{~m}$ ) when compared to the solutions existing in the literature. The effectiveness of the proposed approach has been successfully proven by indoor and outdoor tests, thus leading to the attractive possibility of creating multipurpose and adaptive low cost radar systems to be applied in different contexts.

\section{Conclusions and Future Works}

A low cost, flexible, compact, and versatile solution to create an L-Band SDRadar system has been proposed in this work. The USRP NI2920 platform has been adopted for the first time to realize a SDRadar system for target detection. The main result of integrating NI2920 in the proposed SDRadar system is related to the significant enhancing of slant-range resolution $(6 \mathrm{~m})$ with respect to that achieved in the existing SDRadar solutions. A specific LabVIEW application has been developed to implement the radar processing algorithm as well as to control the various components of the system. A stretch processing approach has been implemented in the system to fully take advantage of the benefit of the available enhanced bandwidth. Outdoor tests, using a basic version of the proposed SDRadar scheme, have been performed, correct distance measurements between the SDRadar platform and some metallic sheets have been obtained, and the expected slant-range resolution has been achieved. Indoor experimental validations have been also performed to demonstrate the correct working of the entire proposed system, in the case of single and multiple targets. As a future extension of this work, the proposed design will be updated by implementing supplementary signal processing features on the proposed SDRadar; for instance, a Doppler shift frequency analysis will be performed for movement detection, so new functions will be added to the proposed platform, with no changes in the hardware design, in order to create, step by step, a simple, low cost, and multipurpose radar.

\section{Conflict of Interests}

The authors state that there are no financial or competing interests which could influence the validity of the present research.

\section{Acknowledgment}

This work has been carried out under the framework of PON 01_01503 National Italian Project "Landslides Early Warning," financed by the Italian Ministry of University and Research.

\section{References}

[1] T. Debatty, "Software defined RADAR a state of the art," in Proceedings of the 2nd International Workshop on Cognitive Information Processing (CIP '10), pp. 253-257, Brussels, Belgium, June 2010.

[2] Z. Hui, L. Lin, and W. Ke, " $24 \mathrm{GHz}$ software-defined radar system for automotive applications," in Proceedings of the 10th European Conference on Wireless Technology (ECWT '07), pp. 138-141, Munich, Germany, October 2007.

[3] D. Garmatyuk, J. Schuerger, and K. Kauffman, "Multifunctional software-defined radar sensor and data communication system," IEEE Sensors Journal, vol. 11, no. 1, pp. 99-106, 2011.

[4] G. Aloi, A. Borgia, S. Costanzo et al., "Software defined radar: synchronization issues and practical implementation," in Proceedings of the 4th International Conference on Cognitive Radio and Advanced Spectrum Management (CogART '11), Barcelona, Spain, October 2011.

[5] C. Prathyusha, S. N. Sowmiya, S. Ramanathan et al., "Implementation of a low cost synthetic aperture radar using software defined radio," in Proceedings of the 2nd International Conference on Computing, Communication and Networking Technologies (ICCCNT '10), Karur, India, July 2010.

[6] M. Fuhr, M. Braun, C. Sturmz, L. Reichardtz, and F. K. Jondral, "An SDR-based Experimental Setup for OFDM-based radar," in Proceedings of the 7th Karlsruhe Workshop on Software Radio, Karlsruhe, Germany, March 2012.

[7] M. Muller, M. Braun, M. Fuhr, and F. K. Jondral, "A USRP-based testbed for OFDM-based radar and communication systems," in Proceedings of the 22nd Virginia Tech Symposium on Wireless Communications, Blacksburg, Va, USA, June 2012.

[8] V. Fernandes, "Implementation of a RADAR System using MATLAB and the USRP," CSUN ScholarWorks, 2012.

[9] National Instruments Data-sheet, NI USRP-2920, NI USRP2921, http://arabia.ni.com/.

[10] U. Pesovic, D. Gliech, P. Planinsiz, Z. Stamenkovic, and S. Randic, "Implementation of IEEE 802.15.4 transceiver on software defined radio platform," in 20th Telecommunications Forum (TELFOR '12), Belgrade, Serbia, November 2012.

[11] S. Costanzo and A. Costanzo, "Compact slotted antenna for wideband radar applications," Advances in Intelligent Systems and Computing, vol. 206, pp. 989-996, 2013.

[12] S. Costanzo, G. A. Casula, A. Borgia et al., "Synthesis of slot arrays on integrated waveguides," IEEE Antennas and Wireless Propagation Letters, vol. 9, pp. 962-965, 2010.

[13] S. Costanzo, I. Venneri, G. Di Massa, and G. Arriendola, "Hybrid array antenna for broadband millimeter-wave applications," Progress in Electromagnetics Research, vol. 83, pp. 173-183, 2008.

[14] F. Venneri, S. Costanzo, G. Di Massa, and G. Amendola, "Aperture-coupled reflectarrays with enhanced bandwidth features," Journal of Electromagnetic Waves and Applications, vol. 22, no. 11-12, pp. 1527-1537, 2008.

[15] F. Venneri, S. Costanzo, and G. Di Massa, "Tunable reflectarray cell for wide angle beam-steering radar applications," Journal 
of Electrical and Computer Engineering, vol. 2013, Article ID 325746, 7 pages, 2013.

[16] F. Venneri, S. Costanzo, and G. Di Massa, "Design of a reconfigurable reflectarray unit cell for wide angle beam-steering radar applications," Advances in Intelligent Systems and Computing, vol. 206, pp. 1007-1013, 2013.

[17] B. R. Mahafza and A. Z. Elsherbeni, Simulations for Radar Systems Design, Chapman \& Hall/CRC, New York, NY, USA, 1999.

[18] M. Skolnik, Radar Handbook, McGraw Hill, San Francisco, Calif, USA, 3rd edition, 2008. 

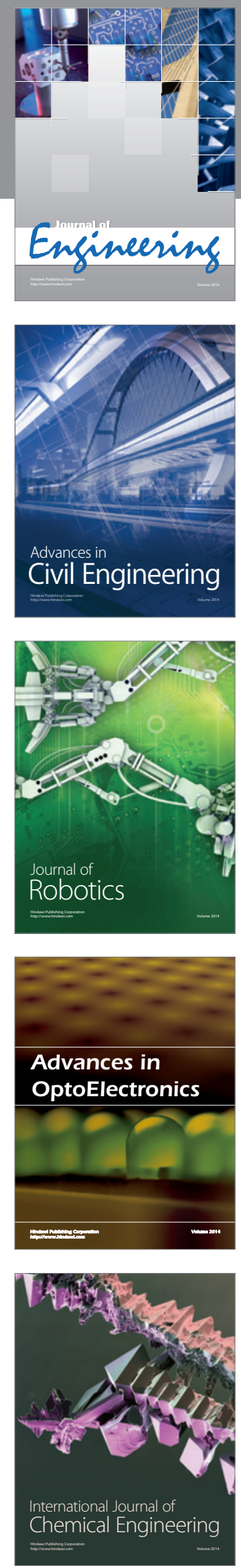

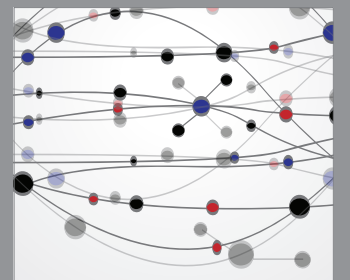

The Scientific World Journal
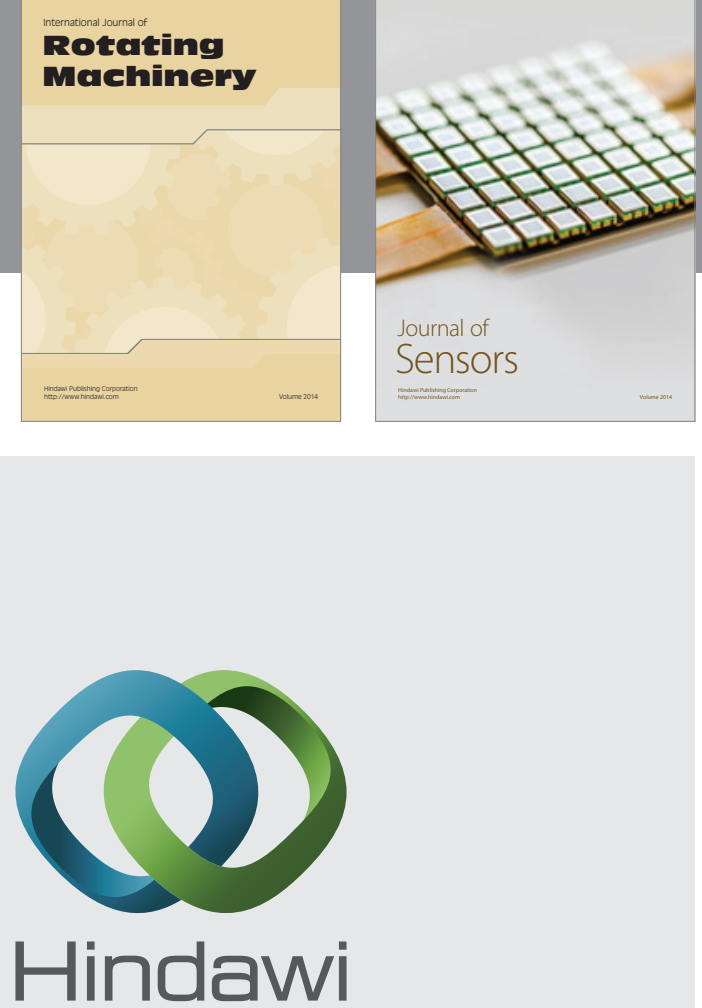

Submit your manuscripts at http://www.hindawi.com
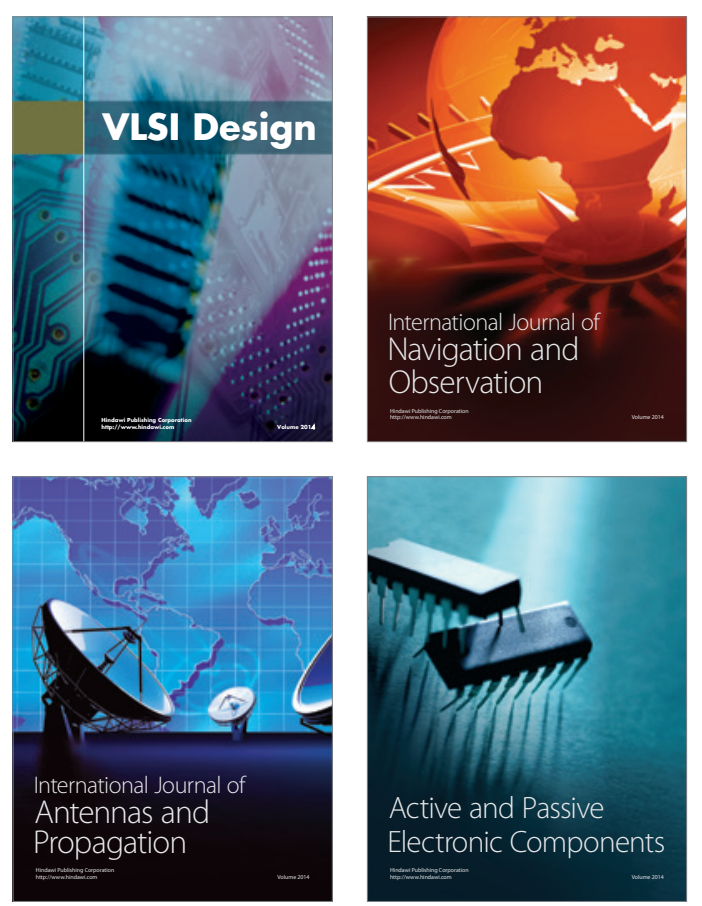
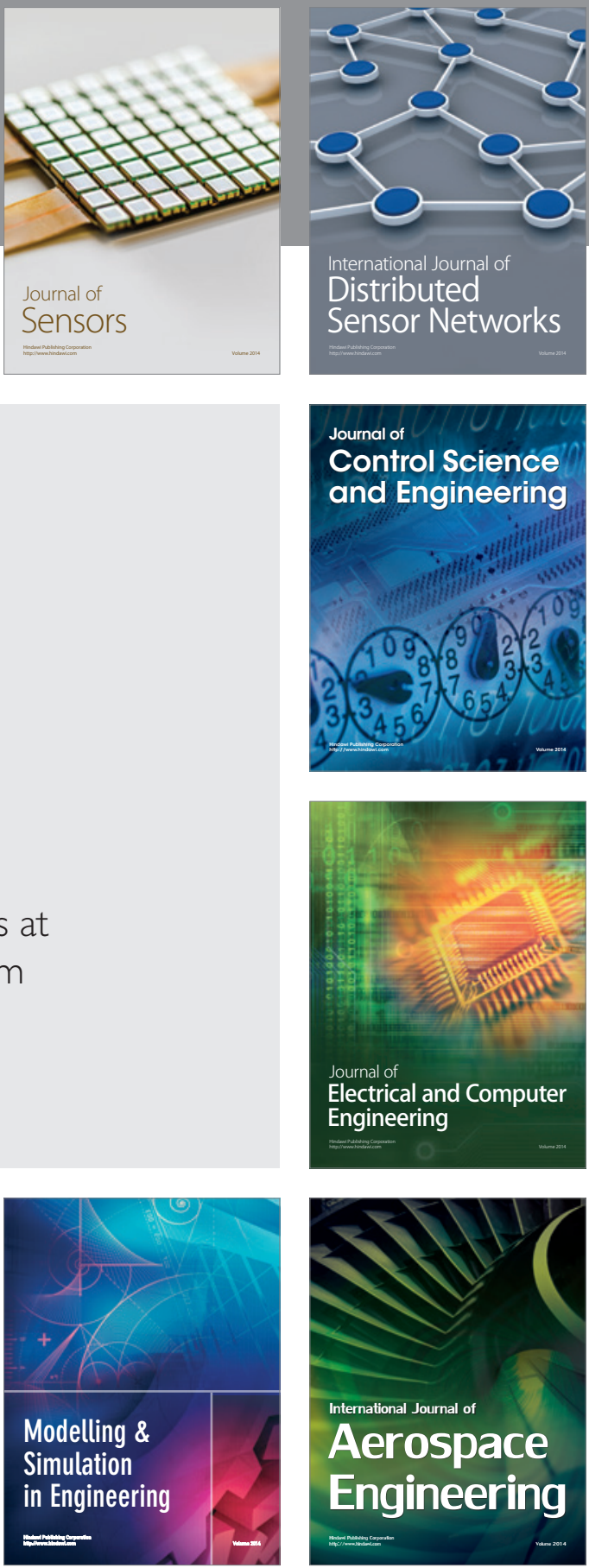

Journal of

Control Science

and Engineering
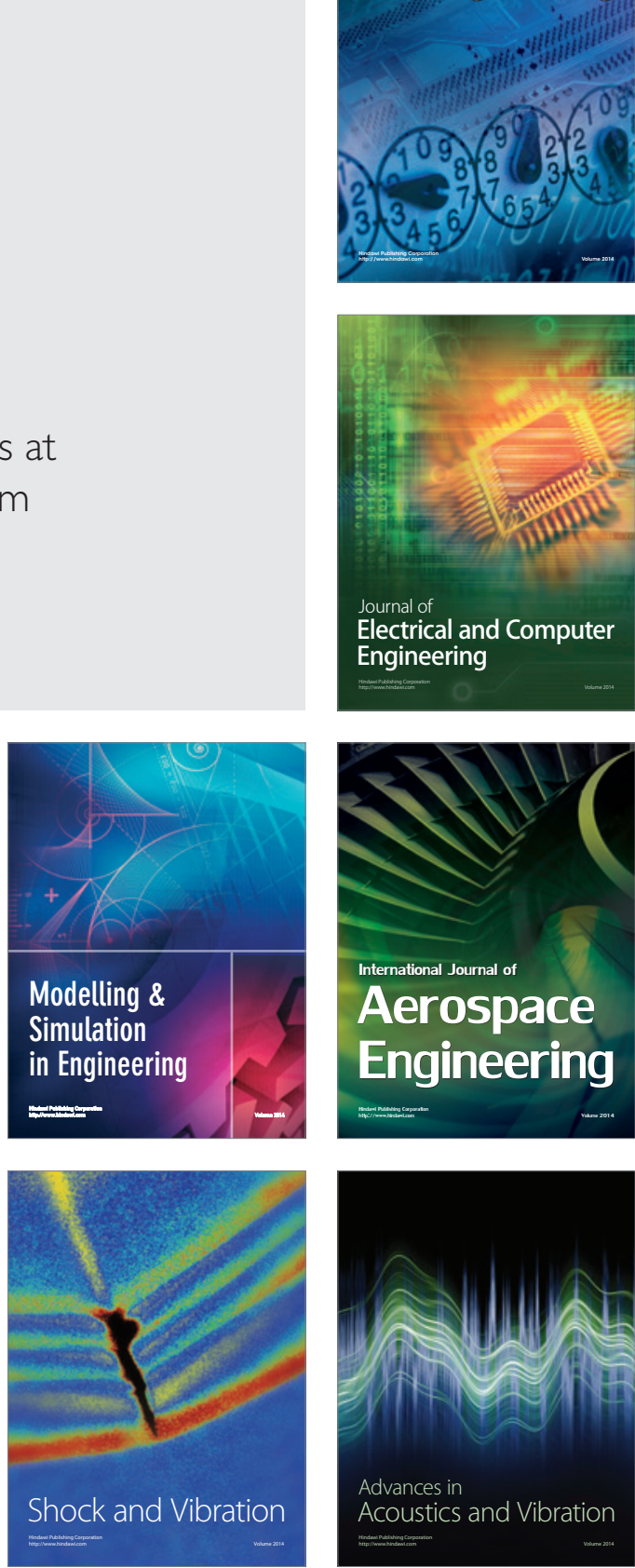